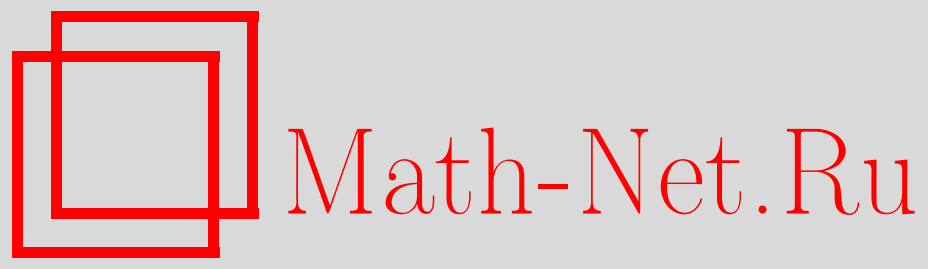

И. М. Кричевер, Коммутирующие разностные операторы и комбинаторное преобразование Гэйла, Функи. анализ и его прил., 2015, том 49, выпуск $3,22-40$

DOI: https://doi.org/10.4213/faa3204

Использование Общероссийского математического портала MathNet.Ru подразумевает, что вы прочитали и согласны с пользовательским соглашением

http://www . mathnet.ru/rus/agreement

Параметры загрузки:

IP : 18.207 .199 .55

26 апреля 2023 г., 11:25:02

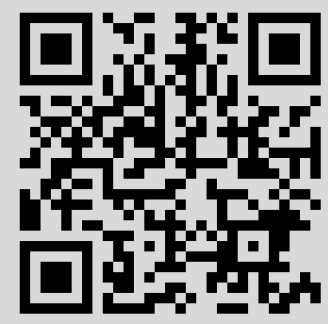




\title{
Коммутирующие разностные операторы и комбинаторное преобразование Гэйла*
}

\author{
(C) 2015. И. М. КРИЧЕВЕР
}

\begin{abstract}
Построена спектральная теория $n$-периодических строго треугольных разностных операторов $L=T^{-k-1}+\sum_{j=1}^{k} a_{i}^{j} T^{-j}$ и спектральная теория «суперпериодических» операторов, для которых все решения уравнения $(L+1) \psi=0$ являются (анти)периодическими. Доказано, что для любого суперпериодического оператора $L$ порядка $k+1$ существует единственный суперпериодический оператор $\mathscr{L}$ порядка $n-k-1$, который коммутирует с $L$, и показано, что дуальность $L \leftrightarrow \mathscr{L}$ с точностью до некоторой инволюции совпадает с комбинаторным преобразованием Гэйла, введенным недавно в $[21]$.
\end{abstract}

\section{$\S 1$. Введение}

В последнее время теория линейных разностных уравнений вида

$$
V_{i}=a_{i}^{1} V_{i-1}-a_{i}^{2} V_{i-2}+\cdots+(-1)^{k-1} a_{i}^{k} V_{i-k}+(-1)^{k} V_{n-k-1}
$$

с вещественными периодическими коэффициентами

$$
a_{i}^{j}=a_{i+n}^{j} \in \mathbb{R}, \quad i \in \mathbb{Z}, 1 \leqslant j \leqslant k,
$$

все решения которых являются $n$-(анти)периодическими,

$$
V_{i+n}=(-1)^{k} V_{i}
$$

привлекла к себе значительное внимание, вызванное связями с теорией $n$-угольников в проективном пространстве (активно исследовавшихся в работах [5], [7], [9], [11]), а также связями с теорией $S L_{k+1}$-фризов (созданной Коксетером и Конвеем в [3], [4] и развитой в работах [1], [2], [19], [20], [22], [26], [27]). Теория фризов является частным случаем теории более общих структур, возникающих в теории билинейного дискретного уравнения Хиротъ (см. работу [31] и содержащиеся в ней ссылки).

Настоящее исследование было мотивировано недавней работой [21], где было доказано, что пространство $\mathscr{E}_{k+1, n}$ уравнений $(1)$, удовлетворяющих дополнительному условию (2), как алгебраическое многообразие изоморфно пространству $\mathscr{F}_{k+1, n}$ так называемых $S L_{k+1}$-фризов ширины $w=n-k-2$, а также было установлено, что если $n$ и $k+1$ взаимно просты, то оба эти пространства изоморфны пространству $\mathscr{C}_{k+1, n}$ классов проективно эквивалентных $n$-угольников в $\mathbb{R P}^{k}$. Основным результатом работы [21] является построение комбинаторного преобразования Гэйла, устанавливающего дуальность пространств $\mathscr{E}_{k+1, n}$ и $\mathscr{E}_{n-k-1, n}$. Это преобразование является аналогом классического преобразования

*Работа выполнена в ИППИ РАН им. Харкевича за счет гранта РНФ (проект № 14-5000150). 
дуальности Гэйла точечных конфигураций в проективных пространствах. Отметим, что как классическое, так и комбинаторное преобразования дуальности Гэйла индуцированы дуальностью грассманианов $\operatorname{Gr}(k+1, n)$ и $\operatorname{Gr}(n-k-1, n)$.

Напомним, что если $\widehat{V}$ является $(n \times(k+1))$-матрицей ранга $k+1$, представляющей точку грассманиана $\operatorname{Gr}(k+1, n)$, то двойственную точку представляет $(n \times(n-k-1))$-матрица $\widehat{W}$, такая, что $\widehat{W}^{T} \widehat{V}=0$. Пространство классов проективно эквивалентных $n$-угольников в $\mathbb{P}^{k}$ является фактором грассманиана по действию тора, $\mathscr{C}_{k+1, n} \simeq \operatorname{Gr}(k+1, n) / \mathbb{T}^{n-1}$ (см. [9]). Точки грассманианов, представляющие двойственные $n$-угольники, связаны соотношением $\widehat{W}^{T} D \widehat{V}=0$, где $D$ - это невырожденная диагональная $(n \times n)$-матрица.

Матрица $\widehat{V}_{(i)}:=\left(V_{i+1}^{(j)}, V_{i+2}^{(j)}, \ldots, V_{i+n}^{(j)}\right)$, строки которой определяются базисными решениями $V^{(j)}$ уравнения $(1)$, задает вложение пространства $\mathscr{E}_{k+1, n}$ В грассманиан $\operatorname{Gr}(k+1, n)$ (зависящее от выбора $i$ ). В работе [21] (предложение 4.3.1) доказано, что определяющим свойством комбинаторной дуальности Гэйла являются соотношения

$$
\widehat{W}_{(i)}^{T} \widehat{\varepsilon} \widehat{V}_{(j)}=0, \quad i-j=n-k-1(\bmod n),
$$

где $\widehat{\varepsilon}$ - это диагональная матрица $(1,-1,1, \ldots)$.

Простейшим примером комбинаторного преобразования Гэйла является Gauss' pentagramma mirificum [8], который осуществляет двойственность между 5-периодическими разностными уравнениями третьего и второго порядков. Точнее, утверждение о двойственности означает, что если все решения уравнения

$$
V_{i}=a_{i} V_{i-1}-b_{i} V_{i-2}+V_{i-3}, \quad a_{i}=a_{i+5}, b_{i}=b_{i+5},
$$

являются 5-периодическими, то все решения уравнения

$$
W_{i}=a_{i} W_{i-1}-W_{i-2}
$$

являются 5 -антипериодическими, $W_{i}=-W_{i+5}$. В терминах $n$-угольников это соответствие сопоставляет классам проективно эквивалентных пятиугольников в $\mathbb{P}^{2}$ классы проективно эквивалентных пятиугольников в $\mathbb{P}^{1}$. Для фризов утверждение Гаусса эквивалентно двойственности между 5-периодическими фризами Коксетера и 5-периодическими $S L_{3}$-фризами.

Целью первой части настоящей работы является введение в уравнение (1) спектралъного параметра и построение спектральной теории Боха-Флоке соответствующих разностных операторов. Следует подчеркнуть, что идея введения спектрального параметра в уравнение (1) сама по себе не нова. В работе [24] для построения полного набора инволютивных интегралов отображения пятиугольника, определенного в [29], спектральный параметр s в уравнение (4) вводился следующим образом:

$$
V_{i}=s a_{i} V_{i-1}-s^{-1} b_{i} V_{i-2}+V_{i-3}, \quad a_{i}=a_{i+5}, b_{i}=b_{i+5} .
$$

Алгебро-геометрическое интегрирование уравнения пятиугольника было проведено в работе [30] с помощью лаксова оператора со спектральным параметром, который приводит к уравнению, калибровочно эквивалентному уравнению (5) (интегрируемость в смысле Лиувилля-Арнольда была доказана в [25]). Аналогичным образом спектральный параметр вводился и в уравнения высших порядков для доказательства алгебро-геометрической интегрируемости высших 
уравнений пятиугольников ([12], [13]; см. также [18]). Следует указать на подход к введению спектрального параметра в уравнение (1), предложенный в работах [6], [10], в основе которого лежат связи с теорией кластерных алгебр и который в случае $S L_{3}$ приводит к уравнению, эквивалентному уравнению (5).

Спектральный параметр $s$ в (5) отражает скалярную инвариантность уравнения пятиугольников (см. [24]). В настоящей работе мы рассматриваем спектральную задачу, отличающуюся от рассмотренных ранее. Точнее, рассмотрим (аффинное) пространство $\mathscr{D}_{k+1, n}$ нормированных, «строго» треугольных $n$-периодических разностных операторов с комплексными (или вещественными) коэффициентами:

$$
\left\{L \in \mathscr{D}_{k+1, n} \mid L=T^{-k-1}+\sum_{j=1}^{k} a_{i}^{j} T^{-j}, a_{i}^{j}=a_{i+n}^{j}\right\} .
$$

Здесь и далее $T$ - это оператор сдвига, действующий на бесконечных последовательностях $\psi=\left\{\psi_{i}\right\}, i \in \mathbb{Z}$, т. е. $(T \psi)_{i}=\psi_{i+1}$. Кроме того, если не оговорено противное, будет предполагаться, что старший коэффициент оператора $L$ всегда не равен нулю,

$$
a_{i}^{1} \neq 0 .
$$

Заметим, что для $E=-1$ уравнение на собственные векторы оператора $L$

$$
L \psi=E \psi
$$

после калибровочного преобразования

$$
V=\varepsilon \psi, \quad \varepsilon:=\left\{\varepsilon_{i}=(-1)^{i}\right\},
$$

совпадает с уравнением (1).

Замечание 1.1. Аналогичным образом спектральный параметр в уравнение (1) мог бы быть введен как коэффициент при последнем члене в правой части уравнения. Однако оба способа введения спектрального параметра на самом деле оказываются эквивалентными и переходят друг в друга при инволюции пространства $\mathscr{D}_{k+1, n}$ :

$$
L \longmapsto L^{\sigma}:=T^{-k-1}\left(L^{*}+1\right)-1,
$$

где $L^{*}$ является формально сопряженным оператором,

$$
L^{*}=T^{k+1}+\sum_{j=1}^{k} T^{j} a_{i}^{j}=T^{k+1}+\sum_{j=1}^{k} a_{i+j}^{j} T^{j} .
$$

После калибровочного преобразования (8) условие (2), определяющее операторы $L \in \mathscr{E}_{k+1, n} \subset \mathscr{D}_{k+1, n}$, приобретает такой вид: все решения $\Psi$ уравнения $(L+1) \Psi=0$ обладают следующим свойством монодромии:

$$
\Psi_{i}=(-1)^{n+k} \Psi_{i-n} .
$$

Для краткости операторы $L \in \mathscr{E}_{k+1, n}$ будем называть в дальнейшем суперпериодическими. 
Замечание 1.2. Выбор мультипликатора $\mu=(-1)^{n+k}$ в (10) соответствует выбору нормировки в рамках несколько более общей постановки задачи: для любой пары комплексных чисел $(e, \mu)$ рассмотрим (аффинное) пространство треугольных $n$-периодических операторов порядка $k+1$, таких, что все решения уравнения $(L-e) \Psi=0$ являются блоховскими с одинаковым мультипликатором $\mu$ :

$$
\mathscr{E}_{k+1, n}^{e, \mu}=\left\{L \in \mathscr{D}_{k+1, n} \mid(L-e) \Psi=0 \Rightarrow \mu \Psi_{i}=\Psi_{i-n}\right\} .
$$

Используя те же аргументы, что и в работе [21], несложно показать, что это пространство непусто тогда и только тогда, когда

$$
\mu^{k+1}=(-1)^{k n} e^{n} .
$$

Действительно, старший коэффициент оператора $L$ равен единице. Следовательно, если $\Psi^{(j)}-$ это набор базисных решений уравнения $(11), \operatorname{to} \operatorname{det} \Phi_{(i)}=$ $(-1)^{k} e^{-1} \operatorname{det} \Phi_{(i-1)}$, где $\Phi_{(i)}:=\left\{\Psi_{i-s}^{(j)}\right\}_{s=0, \ldots, k}^{j=0, \ldots, k}$. Оператор монодромии $T^{-n}$, ограниченный на пространство решений $\Psi^{(j)}$, является скалярным оператором умножения на $\mu$, что влечет за собой с учетом последнего равенства уравнение (12).

Легко видеть, что над комплексными числами все допустимые пары $(e, \mu)$ принадлежат одной орбите действия $(e, \mu) \rightarrow\left(c^{k+1} e, c^{n} \mu\right), c \in \mathbb{C}$. Над вещественными числами можно непосредственно проверить, что если $n$ и $k+1$ взаимно просты, то четность числа $n k$ совпадает с четностью числа $k$ и, как следствие, любая вещественная допустимая пара может быть получена с помощью вещественного преобразования из пары $\left(-1,(-1)^{n+k}\right)$, определяющей суперпериодические операторь.

Скейлинговое преобразование пар чисел, введенное выше, соответствует скейлинговому преобразованию операторов

$$
\tau_{c}: \mathscr{D}_{k+1, n} \longmapsto \mathscr{D}_{k+1, n}, \quad \tau_{c}(L)=c^{k+1} C L C^{-1},
$$

где $C$ является диагональным оператором, $(C \psi)_{i}=c^{i} \psi_{i}$. Отметим, что умножение на $c^{k+1}$ калибровочно преобразованного оператора $C L C^{-1}$ введено для нормировки на единицу старшего коэффициента оператора $\tau_{c}(L)$.

В теории обыкновенных линейных дифференциальных операторов хорошо известно, что если ядро оператора $B$ является подпространством ядра другого оператора $A, \operatorname{ker} B \subset \operatorname{ker} A$, то последний оператор делится на первый, т. е. найдется оператор $C$, такой, что $A=B C$. Это же верно и для разностных операторов. Несложно показать, что если оператор суперпериодичен, то и сопряженный к нему оператор также суперпериодичен. Как следствие получаем, что первоначальное определение суперпериодических операторов эквивалентно следующему операторному условию:

Лемма 1.3. n-периодический треугольный оператор $L \in \mathscr{D}_{k+1, n}$ порядка $k+1$ является суперпериодическим тогда и только тогда, когда оператор $T^{-n}-(-1)^{n+k}$ делится справа и слева на оператор $L+1$, m. е. существуют операторы $L_{r}, L_{l}$ в $\mathscr{D}_{n-k-1, n}$, такие, что выполняются следующие операторные уравнения:

$$
T^{-n}-(-1)^{k+n}=\left(L_{l}-(-1)^{k+n}\right)(L+1)=(L+1)\left(L_{r}-(-1)^{k+n}\right) .
$$


В $\S 3$ будет доказано, что если период $n$ и порядок $k+1$ оператора $L$ взаимно просты, то блоховские решения уравнения (7), т. е. решения этого уравнения, являющиеся собственными для оператора монодромии $T^{n}$,

$$
w \psi_{i}=\psi_{i-n},
$$

совпадают с дискретным аналогом функции Бейкера-Ахиезера, определенной на спектральной кривой оператора $L$. Эта блоховская спектральная кривая отлична от спектральных кривых, возникающих в теории отображения пятиугольника, но, как будет ясно из дальнейшего, связана с комбинаторным преобразованием Гэйла.

Точнее: отождествление блоховских решений уравнения (6) с дискретным аналогом функции Бейкера-Ахиезера позволяет связать спектральную теорию треугольных операторов с теорией коммутирующих разностных операторов ранга 1, построенной в работах [15], [23], и с помощью этой связи прояснить роль определяющего соотношения операторов $L \in \mathscr{E}_{k+1, n}$ и суть комбинаторной двойственности Гэйла.

Появление коммутирующих операторов в теории суперпериодичных разностных операторов является прямым следствием нашего первого результата:

Теорема 1.4. Если числа $n$ и $k+1$ взаимно просты, то

(i) операторы $L_{r}$ u $L_{l}$, определяемые соотношениями (14) для суперпериодического оператора $L$, совпадают, $L_{r}=L_{l}$;

(ii) при инволюиии (9) оператор $\mathscr{L}:=L_{r}=L_{l}$ переходит в оператор, калибровочно эквивалентный оператору $\mathscr{G}(L)$, который Гэйл-двойствен операmopy L:

$$
\mathscr{G}(L)=\varepsilon \mathscr{L}^{\sigma} \varepsilon^{-1} .
$$

Из утверждения (i) теоремы и уравнений (14) вытекает

Следствие 1.5. Операторы $L$ и $\mathscr{L}$ коммутируют друг с другом:

$$
[L, \mathscr{L}]=0 .
$$

Как мы видим, условие существования коммутирующего с ним оператора является необходимым условием того, что периодический треугольный оператор является суперпериодическим. Оказывается, что это условие является не только необходимым, но и достаточным для операторов, которые могут быть получены из суперпериодических с помощью скейлингового преобразования (13).

Теорема 1.6. Предположим, что у оператора $L \in \mathscr{D}_{k+1, n}$, где $2(k+1)<n$, $(n, k+1)=1$, существует коммутирующий с ним оператор $K \in \mathscr{D}_{n-k-1, n}$. Тогда в общем положении

(i) оператор L суперпериодичен с точностью до преобразования (13) с некоторой константой c, m.e. $\tau_{c}(L) \in \mathscr{E}_{k+1, n}$;

(ii) существует единственный полином $P$, такой, что оператор $\mathscr{L}=K+$ $P(L)$ является суперпериодическим с точностью до преобразования (13).

В формулировке утверждения теоремы «общее положение» означает «для открытого по Зарисскому множества в пространстве операторов». Точное описание этого множества будет дано в ходе доказательства теоремы в $§ 4$. 


\section{§2. Дискретная функция Бейкера-Ахиезера}

Для начала напомним основные факты теории коммутирующих разностных операторов ранга 1.

Рассмотрим гладкую алгебраическую кривую $Г$ рода $g$. Зафиксируем на ней две произвольные точки $p_{ \pm} \in \Gamma$ и выберем эффективный дивизор $D=$ $\gamma_{1}+\cdots+\gamma_{g}$ степени $g$. По теореме Римана-Роха для дивизора $D$ общего положения размерность $h^{0}\left(D+i\left(p_{+}-p_{-}\right)\right)$равна 1 для любого $i \in \mathbb{Z}$. Обозначим через $\psi_{i}(p), p \in \Gamma$, единственное сечение пространства $H^{0}\left(D+i\left(p_{+}-p_{-}\right)\right)$. Другими словами, $\psi_{i}$ - это единственная с точностью до умножения на число мероморфная функция, такая, что вне отмеченных точек она имеет полюсы только в точках $\gamma_{s}$ кратности, не превосходящей кратности, с которой $\gamma_{s}$ входит в $D$, а в точке $p_{+}$(соответственно $\left.p_{-}\right)$функция $\psi_{i}$ имеет нуль (полюс) порядка $i$.

Если зафиксировать локальные координаты $z$ в окрестностях отмеченных точек, то лорановские разложения функции $\psi_{i}(p)$ в этих точках имеют вид

$$
\psi_{i}=z^{ \pm i}\left(\sum_{s=0}^{\infty} \xi_{s}^{ \pm}(i) z^{s}\right), \quad z=z(p), p \rightarrow p_{ \pm} .
$$

Функция $\psi:=\psi_{i}(p)$ дискретной переменной $i$ и точки $p$ на кривой $Г$ называется дискретной функцией Бейкера-Ахиезера [15]. Всюду в дальнейшем будет предполагаться, что $\psi$ нормирована условием $\xi_{0}^{+}=1$. Отметим, что при изменении выбора локальной координаты $z \rightarrow \tilde{z}=c z+O\left(z^{2}\right)$ в окрестности точки $p_{+}$нормализованная функция Бейкера-Ахиезера преобразуется следующим образом:

$$
\tilde{\psi}_{i}(p)=c^{i} \psi_{i}(p)
$$

(ср. со скейлинговым преобразованием (13).

Обозначим через $\mathscr{A}\left(p_{+}, p_{-}\right)$кольцо мероморфных функций на кривой $\Gamma$, которые голоморфны вне отмеченных точек $p_{ \pm}$. Из единственности функции Бейкера-Ахиезера непосредственно следует

Лемма 2.1 [15]. Для каждой функиии $A \in \mathscr{A}\left(p_{+}, p_{-}\right)$существует единственный разностный оператор

$$
L_{A}=\sum_{j=-k_{-}}^{k_{+}} a_{i}^{j} T^{-j} \Longleftrightarrow\left(L_{A} \psi\right)_{i}=\sum_{j=k_{-}}^{k_{+}} a_{i}^{j} \psi_{i-j},
$$

maкой, что

$$
L_{A} \psi=A \psi \text {. }
$$

Здесъ $k_{ \pm}$- порядки полюсов функиии $A$ в отмеченных точках $p_{ \pm}$.

Следствие 2.2. Операторы $L_{A}$ коммутируют друг с другом, т.е. $\left[L_{A}, L_{B}\right]$ $=0$.

Коэффициенты оператора $L_{A}$ определяются последовательно из системы уравнений, полученных подстановкой лорановских разложений функций $\psi$ и $A$ около отмеченных точек. Например, если разложение функции $A$ около точки $p_{+}$имеет вид $A=c_{0} z^{-k_{+}}+c_{1} z^{-k_{+}+1}+\ldots$, то

$$
a_{i}^{k_{+}}=c_{0}, \quad a_{i}^{k_{+}-1}=c_{0}\left(\xi_{1}^{+}\left(i-k_{+}\right)-\xi_{1}^{+}(i)\right)-c_{1}, \quad \ldots .
$$


Аналогично, если разложение функции $A$ в окрестности точки $p_{-}$имеет вид $A=c_{0}^{-} z^{-k_{-}}+\ldots$, то

$$
a_{i}^{-k_{-}}=\frac{c_{0}^{-} \xi_{0}^{-}(i)}{\xi_{0}^{-}\left(i+k_{-}\right)}, \ldots
$$

Замечание 2.3. Отметим для использования в дальнейшем, что если $A$ принадлежит подкольцу $\mathscr{A}_{+}\left(p_{+}, p_{-}\right) \subset \mathscr{A}\left(p_{+}, p_{-}\right)$мероморфных функций на $\Gamma$, имеющих единственный полюс в точке $p_{+}$и обращающихся в нуль в точке $p_{-}$, то соответствующий оператор $L_{A}$ является строго треугольным. В этом случае формула (19) для старшего коэффициента $a_{i}^{1}$ принимает вид

$$
a_{i}^{1}=e^{x_{i}-x_{i-1}+l}, \quad x_{n}:=\ln \xi_{0}^{-}(i), l:=\ln c_{0}^{-} .
$$

Замечание 2.4. Описанное выше соответствие продолжается и на сингулярные кривые. Точнее ([15], [23]), существует естественное соответствие

$$
\mathscr{A} \longleftrightarrow\left\{\Gamma, p_{ \pm}, \mathscr{F}\right\}
$$

между кольцами $\mathscr{A}$ коммутирующих линейных разностных операторов, содержащими пару операторов взаимно простых порядков, и наборами алгеброгеометрических данных $\left\{\Gamma, p_{ \pm}, \mathscr{F}\right\}$, где $\Gamma$ - алгебраическая кривая с парой отмеченных гладких точек, а $\mathscr{F}$ - пучок без кручения на $\Gamma$ ранга 1 , такой, что

$$
h^{0}\left(\Gamma, \mathscr{F}\left(i p_{+}-i p_{-}\right)\right)=h^{1}\left(\Gamma, \mathscr{F}\left(i p_{+}-i p_{-}\right)\right)=0 .
$$

Соответствие $(20)$ становится взаимно однозначным, если кольца $\mathscr{A}$ коммутирующих операторов рассматриваются с точностью до сопряжения $\mathscr{A}^{\prime}=g \mathscr{A}^{-1}$, $g=\left\{g_{i}\right\}$ (в своей окончательной форме это соответствие было сформулировано Мамфордом.

Дискретная функция Бейкера-Ахиезера и, как следствие, коэффициенты соответствующих разностных операторов допускают явное выражение в терминах римановых тэта-функций

$$
\theta(z):=\theta(z \mid B)=\sum_{m \in \mathbb{Z}^{g}} e^{2 \pi i(m, z)+\pi i(B m, m)}, \quad z \in \mathbb{C}^{g},
$$

определяемых матрицей $B$, построенной по $b$-периодам нормированных голоморфных дифференциалов $d \omega_{j}$ на $\Gamma$ :

$$
B_{j, k}=\oint_{b_{k}} d \omega_{j}, \quad \delta_{j, k}=\oint_{a_{k}} d \omega_{j} .
$$

Лемма 2.5 [15]. Функиия Бейкера-Ахиезера дается формулой

$$
\psi_{i}(p)=r_{i} \frac{\theta(A(p)+i U+Z) \theta\left(A\left(p_{+}\right)+Z\right)}{\theta\left(A\left(p_{+}\right)+i U+Z\right) \theta(A(p)+Z)} e^{i \Omega_{0}(p)} .
$$

3десъ

(а) $\Omega_{0}(p)$ - абелев интеграл, $\Omega_{0}(p)=\int^{p} d \Omega_{0}$, соответствующий нормированному, $\oint_{a_{k}} d \Omega_{0}=0$, мероморфному дифференциалу на $Г$ с простыми полюсами в отмеченных точках $p_{ \pm} c$ вычетами \pm 1 соответственно;

(b) $A(p)$ - преобразование Абеля, т.е. вектор с координатами $A(p)=\int^{p} d \omega_{k}$;

(c) $U-$ это вектор, равный $\left.A\left(p_{+}\right)-A_{(} p_{-}\right)$;

(d) $Z$ - произвольный вектор (отражающий произвольность выбора дивизора полюсов функиии Бейкера-Ахиезера). 
Из формулы (21) следует, что коэффициенты коммутирующих разностных операторов $L_{A}$ являются квазипериодическими функциями переменной $i$. Операторы с периодическими коэффициентами выделяются следующими условиями на алгебро-геометрические данные.

Лемма 2.6. Предположим, что на кривой Г существует мероморфная функиия $w=w(p)$ с единственным полюсом порядка $n$ в точке $p_{+}$, имеющая нуль порядка $n$ во второй отмеченной точке $p_{-}$. Тогда коэфбициенты соответствующих операторов $L_{A}$ являются $n$-периодическими.

Доказательство. Функция $w$, если она существует, единственна с точностью до умножения на константу. Если первый джет локальной координаты в окрестности точки $p_{+}$зафиксирован, то функция $w$ может быть нормирована условием $w=z^{-n}+O\left(z^{-n+1}\right)$. Из единственности функции Бейкера-Ахиезера $\psi_{i}$ следует, что выполняется соотношение (15), следствием которого является утверждение, что оператор $L_{A}$ является $n$-периодическими.

Вещественные операторы. Формула (21) для $i \in C$ определяет $\psi_{i}(p)$ как многозначную функцию на $\Gamma$. Однозначная ветвь $\psi_{i}(p)$ может быть определена на $Г$ с разрезом между отмеченными точками, который не пересекает базисные $a$ - и $b$-циклы. Коэффициенты $a_{i}^{j}$ соответствующих операторов являются мероморфными функциями комплексной переменной $i$. Спектральные данные, приводящие к операторам с вещественными (при $i \in \mathbb{R}$ ) коэффициентами, выделяются следующим образом:

Лемма 2.7. Предположим, что на алгебраической кривой $\Gamma$ с двумя отмеченными точками $p_{ \pm}$существует антиголоморфная инволюиия $\tau$, для которой отмеченные точки неподвижны, $\tau\left(p_{ \pm}\right)=p_{ \pm}$. Тогда функиия БейкераАхиезера $\psi$, соответствующая вещественному дивизору $D=\tau(D)$, удовлетворяет соотношению

$$
\psi(p)=\bar{\psi}(\tau(p))
$$

(в предположении, что координата z в окрестности точки $p_{+}$, используемая для нормировки функции $\psi$, также вещественна, $\left.\bar{z}=\tau^{*} z\right)$.

Из уравнения (22) следует, что если функция $A$ вещественна, $A=\bar{A}(\tau(p))$, то и коэффициенты соответствующего оператора $L_{A}$ являются вещественными (возможно, сингулярными) функциями переменной $i \in \mathbb{R}$. В рамках теории конечнозонного интегрирования известны два типа условий, гарантирующих регулярность коэффициентов операторов. Изложим тот из них, который отвечает рассматриваемой нами ситуации.

Для этого напомним, прежде всего, что голоморфная антиинволюция $\tau$ гладкой алгебраической кривой $\Gamma$ рода $g$ имеет не более чем $g+1$ неподвижных овалов. Кривые с максимально возможным числом неподвижных овалов $A_{0}, \ldots, A_{g}$ называются $M$-кривъмии.

Лемма 2.8. Предположим, что кривая Г является $M$-кривой с отмеченными точками на одном из неподвижнных овалов, $p_{ \pm} \in A_{0}$. Предположим также, что точки дивизора $D$ выбраны по одной на каждом из оставиихся неподвижных овалов, $\gamma_{s} \in A_{s}, s=1, \ldots, g$. Тогда для любой вещественной функиии $A=\bar{A}(\tau(p)) \in \mathscr{A}\left(p_{+}, p_{-}\right)$коэффиииенты операторов $L_{A}$, соответствующих функиии Бейкера-Ахиезера, построенной по дивизору $D$, являются 
вещественными и неособыми. Более того, стариие коэфбициенты этих операторов $a_{i}^{-k_{-}}$знакоопределены:

$$
a_{i}^{k_{+}}=c_{0}, \quad \operatorname{sgn}\left(a_{i}^{-k_{-}}\right)=\operatorname{sgn} c_{0}^{-} .
$$

Доказательство леммы стандартно в конечнозонной теории (см., например, доказательство аналогичного утверждения для разностного оператора Шрёдингера в [16]).

В дальнейшем мы воспользуемся еще одним основным понятием теории алгебро-геометрического интегрирования.

Двойственная функция Бейкера-Ахиезера. Понятие двойственной функции Бейкера-Ахиезера $\psi^{+}$является универсальным и лежит в основе понятия билинейной формы Хироты солитонных уравнений. В дискретном случае двойственная функция Бейкера-Ахиезера задается двойственным дивизором $D^{+}=\gamma_{1}^{+}+\cdots+\gamma_{g}^{+}$, который определяется из уравнения

$$
D+D^{+}=K+p_{-}+p_{+} \in J(\Gamma)
$$

где $K$ - канонический класс. Другими словами, точки дивизоров $D$ и $D^{+}$являются нулями мероморфного дифференциала $d \Omega$ с простыми полюсами в $p_{ \pm}$с вычетами \pm 1 соответственно. Двойственная функция Бейкера-Ахиезера определяется следующими аналитическими свойствами:

(i) функция $\psi_{i}^{+}$(как функция переменной $p \in \Gamma$ ) вне точек $p_{ \pm}$имеет не более чем простые полюсы в точках $\gamma_{1}^{+}, \ldots, \gamma_{g}^{+}$(если все они различны);

(ii) в окрестности точек $p_{ \pm}$функция $\psi^{+}$имеет вид

$$
\psi_{i}^{+}=z^{\mp i}\left(\sum_{s=0}^{\infty} \chi_{s}^{ \pm}(i) z^{s}\right), \quad z=z(p), p \rightarrow p_{ \pm}, \chi_{0}^{+}=1 .
$$

Фактически $\psi^{+}$является функцией того же самого типа, что и сама функция Бейкера-Ахиезера, и поэтому допускает тэта-функциональное представление того же самого типа:

$$
\psi_{i}^{+}(p)=r_{i}^{+} \frac{\theta\left(A(p)-i U-Z-A\left(p_{+}\right)-A\left(p_{-}\right)\right) \theta\left(A\left(p_{-}\right)+Z\right)}{\theta\left(A\left(p_{-}\right)+i U+Z\right) \theta\left(A(p)-Z-A\left(p_{+}\right)-A\left(p_{-}\right)\right)} e^{-i \Omega_{0}(p)},
$$

Лемма 2.9. Пусть $\psi-\oint у н к и и я ~ Б е и ̆ к е р а-А х и е з е р а ~ и ~ \psi^{+}-$двойственная $\kappa$ ней функиия. Тогда имеют место равенства

$$
\operatorname{res}_{p_{+}}\left(\psi_{i}^{+} \psi_{j}\right) d \Omega=\delta_{i, j}, \quad i, j \in \mathbb{Z}
$$

Из определения дуальности следует, что дифференциал в левой части равенства (23) при $i \neq j$ имеет полюс только в одной отмеченной точке $p_{ \pm}$. Следовательно, он не имеет вычета. Дифференциал $\psi_{i}^{+} \psi_{i} d \Omega$ имеет простые полюсы в точках $p_{+}$и $p_{-}$с вычетами \pm 1 соответственно. Лемма доказана.

Следствие 2.10. Пусть $\psi$ является функиией Бейкера-Ахиезера, и пусть $L_{A}$ - это задаваемый ею с помощью уравнения (18) линейный оператор. Тогда двойственная функиия Бейкера-Ахиезера является решением бормально сопряэсенного уравнения

$$
\psi^{+} L_{A}=A \psi^{+}
$$

Напомним, что правое действие разностного оператора определяется как формально сопряженное действие, т. е. $f^{+} T=T^{-1} f^{+}$. 


\section{§3. Теория Блоха-Флоке треугольных периодических разностных операторов}

Сначала кратко напомним стандартную схему построения спектральной теории периодических операторов. Рассмотрим $n$-периодический линейный разностный оператор $L$. Оператор монодромии $T^{-n}$ оставляет инвариантным $(k+1)$-мерное пространство $\mathscr{L}(E)$ решений уравнения $L \psi=E \psi$. Обозначим через $T^{-n}(E)$ ограничение оператора $T^{-n}$ на пространство $\mathscr{L}(E)$. Совместные собственные значения оператора $L$ и оператора монодромии удовлетворяют алгебраическому соотношению

$$
R(w, E)=\operatorname{det}\left(w \cdot 1-T^{-n}(E)\right)=0 .
$$

То же самое уравнение можно получить, если рассмотреть $n$-мерное пространство $\mathscr{T}(w)$ решений уравнения $(15)$ и обозначить через $L(w)$ ограничение оператора $L$ на это пространство $\mathscr{T}(w)$. Тогда

$$
R(w, E)=\operatorname{det}(L(w)-E \cdot 1)=0 .
$$

Наше первое наблюдение, оказавшееся решающим для дальнейшего, относится к специальному виду полинома $R$ и вытекающим из него свойствам спектральной кривой.

Лемма 3.1. Если $n$ и $k+1$ взаимно просты, то спектральная кривая оператора $L \in \mathscr{D}_{k+1, n}$ задается уравнением вида

$$
R(w, E)=w^{k+1}-E^{n}+\sum_{i>0, j \geqslant 0, n i+(k+1) j<n(k+1)} r_{i j} w^{i} E^{j}=0,
$$

где

$$
r:=r_{1,0}=\prod_{j=1}^{n} a_{j}^{1} .
$$

Замечание 3.2. Утверждение леммы - это утверждение, что многоугольник Ньютона полинома $R$ является треугольником с вершинами $(0, n),(k+1,0)$, $(1,0)$, дополненное явными выражениями для коэффициентов при членах, отвечающих вершинам треугольника. Прежде чем представить доказательство леммы, остановимся на специальных свойствах алгебраических кривых, заданных уравнением (24).

Из (24) следует, что аффинная кривая, заданная уравнением $R(w, E)=0$, в бесконечности компактифицируется одной гладкой точкой $p_{+}$. В этой точке функция $w=w(p), p \in \Gamma$, имеет полюс порядка $n$, а функция $E=E(p)$ имеет полюс порядка $k+1$. Из уравнения (24) также следует, что если $w=0$, то $E=0$. Более того, если $r \neq 0$, то одна ветвь многозначной функции $w(E)$, заданной уравнением (24), в окрестности начала координат имеет вид

$$
w=r^{-1} E^{n}\left(1+\sum_{s=1}^{\infty} v_{s} E^{s}\right) .
$$

Следовательно, на кривой Г имеется еще одна выделенная гладкая точка $p_{-}$, в которой функция $w$ имеет нуль порядка $n$ и $E$ имеет простой нуль. Поскольку степени дивизоров нулей и полюсов равны, функция $w$ нигде не равна нулю на $\Gamma \backslash p_{-}$. 
Обозначим через $\mathscr{S}_{k+1, n}$ семейство кривых $\Gamma$, задаваемых уравнением (24). Его размерность (число параметров, т. е. коэффициентов $r_{i j}$ ) равна $k(n+1) / 2$. Для общих значений параметров $r_{i j}$ кривая $\Gamma$ является гладкой алгебраической кривой, род которой равен $g=k(n-1) / 2$. Обозначим через $\mathscr{P}_{k+1, n}$ расслоение якобианов над открытой частью семейства $\mathscr{S}_{k+1, n}$, отвечающей гладким спектральным кривым. Любая точка общего положения $\{\Gamma, D\}$ этого расслоения определяет функцию Бейкера-Ахиезера $\psi$, а затем, как следствие леммы 2.1, определяет оператор $L_{E}$, соответствующий функции $E=E(p)$. Эта функция имеет полюс порядка $k+1$ в точке $p_{+}$и равна нулю во второй отмеченной точке $p_{-}$. Следовательно, оператор $L_{E}$ является строго нижнетреугольным. Более того, в силу следствия 2.6 оператор $L_{E}$ является $n$-периодическим. Установленное соответствие открытой области

$$
\mathscr{P}_{k+1, n}^{0} \subset \mathscr{P}_{k+1, n} \longmapsto \mathscr{D}_{k+1, n}
$$

будет называться обратным спектральным преобразованием.

Пример. Для $k=1, n=2 m+1$ кривые семейства $\mathscr{S}$ являются гиперэллиптическими кривыми, заданными уравнением вида

$$
w^{2}+Q_{m}(E) w-E^{2 m+1}=0,
$$

где $Q_{m}$ является полиномом степени $m$.

Доказательство леммы 3.1. По определению полином $R$ имеет степень $k+1$ по переменной $w$ и степень $n$ по переменной $E$. Матрица $L(w)$ содержит $k+1$ ненулевых диагоналей под главной диагональю и $k+1$ диагоналей в верхнем правом углу. Элементы последних диагоналей содержат в качестве множителя переменную $w$. Следовательно, $R(0, E)=E^{n}$ и суммирование в $(24)$ проводится только по $i>0$. Коэффициент $r_{1,0}$ равен произведению элементов матрицы на первой под главной диагональю диагонали и коэффициента при $w$ в правом верхнем углу матрицы. Это доказывает равенство (25).

Для завершения доказательства леммы осталось лишь доказать, что суммирование в $(24)$ проводится по индексам $(i, j)$, таким, что $n i+j(k+1)<$ $n(k+1)$. Для использования в дальнейшем мы приведем доказательство последнего утверждения, аналогичное тому, которое использовалось в теории коммутирующих разностных операторов (см. подробности в [17]).

Лемма 3.3. Если числа п и $k+1$ взаимно прость, то для любого оператора $L \in \mathscr{D}_{k+1, n}$ существует единственный формальный ряд

$$
E(z)=z^{-(k+1)}\left(1+\sum_{s=1}^{\infty} e_{s} z^{s}\right),
$$

такой, что уравнение $L \psi=E \psi$ имеет единственное решение

$$
\psi_{i}(z)=z^{i}\left(1+\sum_{s=1}^{\infty} \xi_{s}^{+}(i) z^{s}\right)
$$

с периодическими коэффичиентами

$$
\xi_{s}^{+}(i)=\xi_{s}^{+}(i+n),
$$

нормированное условием $\xi_{s}^{+}(0)=0$. 
Доказательство. Подстановка рядов $(27)$ и (28) в уравнение $L \psi=E \psi$ дает систему разностных уравнений на их неизвестные коэффициенты $e_{s}$ и $\xi_{s}$. Первым из них является уравнение

$$
e_{1}+\xi_{1}^{+}(i)-\xi_{1}^{+}(i-k-1)=a_{i}^{k} .
$$

Условие периодичности (29) однозначно определяет $e_{1}=n^{-1} \sum_{i=1}^{n} a_{i}^{k}$ и сводит разностное уравнение (30) порядка $k+1$ к разностному уравнению порядка 1 :

$$
m e_{s}+\xi_{1}^{+}(i)-\xi_{1}^{+}(i-1)=\sum_{j=0}^{m-1} a_{i-j(k+1)}^{k},
$$

где $m$ - это целое число, $1 \leqslant m<n$, такое, что $m(k+1)=1(\bmod n)$. Уравнение (31) и начальное условие $\xi_{1}^{+}(0)=0$ однозначно определяют $\xi_{1}^{+}(i)$.

Для произвольного $s$ уравнения на $e_{s}$ и $\xi_{s}^{+}$имеют вид

$$
e_{s}+\xi_{s}^{+}(i)-\xi_{s}^{+}(i-k-1)=Q_{s}\left(e_{1}, \ldots, e_{s-1} ; \xi_{1}, \ldots, \xi_{s-1}\right),
$$

где $Q_{s}$ - некоторое явное выражение, линейное по переменным $e_{s^{\prime}}, \xi_{s^{\prime}}, s^{\prime}<s$, и полиномиально зависящее от коэффициентов оператора $a_{i}^{j}$. Те же аргументы, что и использованные выше, доказывают что они имеют единственное периодическое решение. Лемма доказана.

По определению ряд $E(z)$ является формальным рядом. Оказывается, что он является сходящимся и представляет собой ряд Пюизо функции $E$, рассматриваемой как аналитическая функция на алгебраической кривой, заданной уравнением (24), в окрестности бесконечности. Точнее, аргументы, идентичные тем, которые использовались в [14] (см. детали в [17]), позволяют доказать следующую лемму.

Лемма 3.4. Пусть $E(z)$ - формальный ряд, определенный в силу леммы 3.3. Тогда характеристический полином $R(w, E)$ - это

$$
R(w, E)=\prod_{j=1}^{n}\left(E-E\left(z_{j}\right)\right), \quad z_{j}^{-n}=w .
$$

Правая часть равенства (32) является симметрической функцией переменных $z_{j}$. Следовательно, априори она является полиномом по переменной $E$ и (формальным) лорановским рядом по переменной $w^{-1}$. Уравнение (32) утверждает, что этот ряд на самом деле является полиномом по переменной $w$. Кроме того, следствием этого уравнения является то, что степень по переменной $z$ всех членов полинома $R(w, E)$ кроме $w^{k+1}$ и $E^{n}$ строго меньше, чем $n(k+1)$. Лемма 3.1 доказана.

Аналогичным образом описываются блоховские решения в окрестности второй отмеченной точки.

Лемма 3.5. Для любого строго треугольного (не обязательно периодического) оператора $L$ уравнение $L \psi=E \psi$ имеет единственное формальное решение вида

$$
\psi_{i}(E)=e^{x_{i}} E^{i}\left(1+\sum_{s=1}^{\infty} \xi_{s}^{-}(i) E^{s}\right), \quad e^{x_{i}-x_{i-1}}:=a_{i}^{1},
$$

нормированное условием $\xi_{s}^{-}(0)=0$.

2 Функциональный анализ и его приложения, т. 49, вып. 3 
Доказательство. Подстановка ряда (33) в уравнение (7) дает систему уравнений на неизвестные коэффициенты $\xi_{s}^{-}$. Они являются неоднородными линейными разностными уравнениями первого порядка

$$
\xi_{s}^{-}(i)-\xi_{s}^{-}(i-1)=q_{s}\left(\xi_{1}^{-}, \ldots, \xi_{s-1}^{-}\right),
$$

которые при заданных начальных условиях позволяют однозначно восстановить $\xi_{s}^{-}(i)$ для всех $i$.

Из единственности формального решения (33) вытекает

Следствие 3.6. Для $L \in \mathscr{D}_{k+1, n}$ формальное решение (33) является блоховским решением, т.е. удовлетворяет уравнению (15), где

$$
w(E)=\psi_{n}(E)=E^{n} \sum_{s=0}^{\infty} w_{s} E^{s} .
$$

Координаты блоховского решения $\psi_{i}=\psi_{i}(p), p=(w, E) \in \Gamma$, нормированного условием $\psi_{0} \equiv 0$, являются рациональными функциями переменных $w, E$. Стандартные аргументы теории конечнозонного интегрирования показывают, что полюсы функций $\psi_{i}$ не зависят от $i$. Для того чтобы найти степень дивизора полюсов $D$, достаточно, как обычно, рассмотреть матрицу $F^{i j}=\psi_{i}\left(p_{j}(E)\right)$, $0 \leqslant i \leqslant k$, где $p_{j}(E)=\left(w_{j}, E\right), 1 \leqslant j \leqslant k+1$, - это прообразы точки $E$ на $\Gamma$ при проекции $p \in \Gamma \rightarrow E(p) \in \mathbb{C}$. Матрица $F$ зависит от упорядочивания прообразов, но квадрат ее детерминанта $f(E)=\operatorname{det}^{2} F(E)$ не зависит от него, т. е. $f$ является рациональной функцией переменной $E$. Она имеет двойные полюсы в проекциях полюсов функции $\psi$ (если они различны) и полюс порядка $2 k$ в точке $E=0$. Если кривая Г гладкая, то функция $f$ обращается в нуль только в образах конечных точек ветвления накрытия $E: \Gamma \rightarrow \mathbb{C}$. При этом кратность нуля равна кратности ветвления. Бесконечная точка $p_{+}$ является точкой ветвления накрытия $\Gamma \rightarrow \mathbb{C}$ кратности $k$. Из разложения $(28)$ следует, что в бесконечности, $E=\infty$, функция $f$ имеет нуль порядка $k$. Степени дивизоров полюсов и нулей рациональной функции равны. Следовательно, $2 \operatorname{deg} D+2 k=\nu$, где $\nu$ равно суммарной кратности ветвления накрытия. Степень накрытия $\Gamma \rightarrow \mathbb{C}$ равна $k+1$. По формуле Гурвица род кривой $\Gamma$ (если она гладкая) равен $2 g=\nu-2 k$. Следовательно, $\operatorname{deg} D=g$ и, значит, мы доказали, что блоховские решения на гладких спектральных кривых совпадают с дискретной функцией Бейкера-Ахиезера.

Замечание 3.7. Коэффициенты $r_{i j}$ характеристического уравнения (24) являются полиномиальными функциями от коэффициентов оператора $L \in \mathscr{D}_{k+1, n}$. Прямое доказательство того, что они являются независимыми, весьма нетривиально. Автору не известно никакого другого универсального способа доказательства их независимости, кроме использования прямого и обратного спектральных преобразований. В рамках этого подхода для доказательства независимости достаточно доказать что образ соответствия $\mathscr{D}_{k+1, n} \rightarrow \mathscr{S}_{k+1, n}$, содержит по крайней мере одну гладкую спектральную кривую. А последнее утверждение устанавливается построением обратного спектрального преобразования (26).

Объединяя результаты, доказанные выше, мы приходим к следующему утверждению. 
Теорема 3.8. В предположении, что числа $n$ и $k+1$ взаимно просты, отображение (26) устанавливает взаимно однозначное соответствие между открытой по Зарисскому областью расслоения якобианов над семейством спектральных кривых, заданных уравнением (24), и открытой областью пространства $\mathscr{D}_{k+1, n}$ n-периодических строго нижснетреугольных разностных операторов порлдка $k+1$.

\section{§4. Суперпериодические разностные операторы}

В этом параграфе мы рассмотрим спектральную теорию суперпериодических операторов. Спектральная кривая $\Gamma_{\text {spec }}$ суперпериодического оператора $L \in \mathscr{E}_{k+1, n}$ никогда не является гладкой. Из определения пространства $\mathscr{E}_{k+1, n}$ следует, что точка спектральной кривой $p_{0}=\left(w=(-1)^{n+k}, E=-1\right) \in \Gamma_{\mathrm{spec}}$ является кратной точкой порядка $k+1$ (в которой все листы накрытия $E: \Gamma_{\mathrm{spec}} \rightarrow$ $\mathbb{C}$ пересекаются трансверсально). Последнее условие требует обращения в нуль всех членов тейлоровского разложения полинома $R$ в окрестности точки $p_{0}$, порядки которых меньше, чем $k$. Это дает $(k+1)(k+2) / 2$ линейных уравнений на коэффициенты полинома $R$. Между этими уравнениями имеется одно соотношение, поскольку если число $w=(-1)^{n+k}$ является корнем уравнения $R(w,-1)=0$ кратности по крайней мере $k$, то оно является корнем кратности $k+1$. Следовательно, размерность пространства $\mathscr{S}_{\text {sрес }}$ спектральных кривых операторов $L \in \mathscr{E}_{k+1, n}$ равна $k(n+1) / 2-(k+1)(k+2) / 2+1=k(n-k-2) / 2$.

Обозначим через $\Sigma_{k+1, n}$ пространство алгебраических кривых $\Gamma$, являющихся частичной нормализацией $\pi: \Gamma \rightarrow \Gamma_{\text {spec }}$ спектральных кривых, полученных регуляризацией кратной точки $p_{0}$, т. е. отображение $\pi$ взаимно однозначно вне множества $k+1$ гладких точек $p^{j} \in \Gamma, j=1, \ldots, k+1$, которые являются прообразами точки $p_{0}, \pi\left(p^{j}\right)=p_{0}$. Другими словами, гладкая кривая $Г$ из семейства $\Sigma_{k+1, n}$ может быть охарактеризована следующими условиями:

(i) на кривой Г существует мероморфная функиия $w$ с полюсом порядка $n$ в точке $p_{+}$и нулем порядка $n$ во второй отмеченной точке $p_{-}$;

(ii) на кривой $Г$ существует мероморфная функиия $E$ с полюсом порядка $k+1$ в точке $p_{+}$и такал, что нули $p^{j}$ функиии $E+1$ различны и, более того, в этих точках функция $w-(-1)^{k+n}$ равна нулю:

$$
E\left(p^{j}\right)=-1, \quad w\left(p^{j}\right)=(-1)^{k+n}, \quad j=1, \ldots, k+1 .
$$

При нормализации $\pi$ арифметический род кривой $\Gamma_{\text {spec }}$ уменьшается на $k(k+1) / 2$. Следовательно, для открытой части пространства $\Sigma_{k+1, n}$, отвечающей спектральным кривым, для которых точка $p_{0} \in \Gamma_{\text {sрес }}$ является единственной сингулярной точкой кривой, род соответствующей гладкой кривой $Г$ равен $g=k(n-1) / 2-k(k+1) / 2=k(n-k-2) / 2$.

Рассмотрим прообраз $\pi^{*}(\psi)$ блоховской функции при отображении нормализации (который мы будем по-прежнему обозначать через $\psi$ ). Он имеет те же разложения (28), (33) в окрестности отмеченных точек $p_{ \pm} \in \Gamma$. Функция $E(p)$, $p \in \Gamma$, задает накрытие $\Gamma \rightarrow \mathbb{C}$, которое имеет $k+1$ непересекающихся листов над точкой $E=-1$. Тот же самый подсчет, что и ранее, показывает, что функция $\psi$ имеет $g$ полюсов на кривой $Г$. Тем самым мы приходим к следующему утверждению: 
Теорема 4.1. Для открытого по Зарисскому множества операторов $L \in$ $\mathscr{E}_{k+1, n}$ соответствующие блоховские решения $\psi$ уравнения $L \psi=E \psi$ nараметризуются точками р гладкой алгебраической кривой $\Gamma \in \Sigma_{k+1, n}$. Более того, функиия $\psi_{i}(p)$ является функиией Бейкера-Ахиезера.

Отметим, что подстановка в формулу (21) точек $p^{j} \in \Gamma$ дает явные тэта-функциональные формулы для базиса $\Psi^{(j)}:=\psi\left(p^{j}\right)$ решений уравнения $(L+1) \Psi=0$.

Следствие 4.2. Прямое и обратное спектральные преобразования устанавливают взаимно однозначное соответствие

$$
\mathscr{P} \Longleftrightarrow \mathscr{E}_{k+1, n}
$$

между открытым подмножеством расслоения якобианов Р над подпространством гладких кривых из $\Sigma_{k+1, n}$ и открытым подмножеством пространства суперпериодических операторов.

Теперь мы готовы доказать теорему 1.4.

Доказательство теоремы 1.4. Рассмотрим функцию

$$
G=\frac{w-(-1)^{n+k}}{E+1}+(-1)^{n+k}
$$

на $\Gamma \in \Sigma_{k+1, n}$. В нулях $p^{j}$ знаменателя дроби числитель также равен нулю. Следовательно, функция $G(p)$ голоморфна на $\Gamma \backslash p_{+}$. В отмеченной точке $p_{+}$ функция $G$ имеет полюс порядка $n-k-1$ и обращается в нуль в точке $p_{-}$. По лемме 2.1 существует оператор $\mathscr{L}=L_{G} \in \mathscr{D}_{n-k-1, n}$, такой, что $\mathscr{L} \psi=G \psi$, где $\psi$ - это блоховские решения уравнения $L \psi=E \psi$. Из определения функции $G$ следует операторное равенство

$$
T^{-n}-(-1)^{k+n}=\left(\mathscr{L}-(-1)^{k+n}\right)(L+1)=(L+1)\left(\mathscr{L}-(-1)^{k+n}\right) .
$$

Тем самым часть (i) теоремы доказана.

Функция $w-(-1)^{k+n}$ имеет $n$ нулей на кривой Г. Из них $k+1-$ это точки $p^{j}$. Обозначим через $q^{m}, m=1, \ldots, n-k-1$, набор остальных нулей. В этих точках $G\left(q^{m}\right)=(-1)^{k+n}$. Значит, $\mathscr{L} \in \mathscr{E}_{n-k-1, n}$.

Для того чтобы завершить доказательство теоремы, осталось только доказать равенство (16). Последнее является прямым следствием такого утверждения:

Лемма 4.3. Пусть $\widehat{\Psi}_{(i)}$ - матрица размера $n \times(k+1)$ со столбцами

$$
\left(\psi_{i+1}\left(p^{j}\right), \ldots, \psi_{i+n}\left(p^{j}\right)\right)
$$

$u \widehat{\Psi}_{(i)}^{+}-$матрица размера $(n-k-1) \times n$ со строками

$$
\left(\psi_{i+1}^{+}\left(q^{m}\right), \ldots, \psi_{i+n}^{+}\left(q^{m}\right)\right),
$$

где $\psi$ и $\psi^{+}$- это функиия Бейкера-Ахиезера и ее двойственная на $\Gamma \in \Sigma_{k+1, n}$, а точки $\left\{p^{j}, q^{m}\right\}$ являются нулями функции $w-(-1)^{k+n}$. Тогда для любого иелого $і$ выполнены следуюшие соотношения ортогональности:

$$
\widehat{\Psi}_{(i)}^{+} \widehat{\Psi}_{(i)}=0 .
$$


Доказательство. Равенство (34) является следствием соотношений ортогональности собственных векторов и ковекторов линейного оператора, отвечающих различным собственным значениям,

$$
E\left(q^{m}\right)\left\langle\psi^{+}\left(q^{m}\right), \psi\left(p^{j}\right)\right\rangle=\left\langle\psi^{+}\left(q^{m}\right), L \psi\left(p^{j}\right)\right\rangle=E\left(p^{j}\right)\left\langle\psi^{+}\left(q^{m}\right), \psi\left(p^{j}\right)\right\rangle,
$$

где $\langle\cdot, \cdot\rangle$ обозначает естественное спаривание векторов и ковекторов. По определению $E\left(p^{j}\right)=-1$. Степень функции $E$ равна $k+1$. Следовательно, она не равна -1 нигде, кроме точек $p^{j}$, т. е. $E\left(p^{j}\right) \neq E\left(q^{m}\right)$. Учитывая последнее соотношение, получаем, что равенство (35) выполнено, только если выполнено равенство

$$
\left\langle\psi^{+}\left(q^{m}\right) \psi\left(p^{j}\right)\right\rangle=\sum_{s=1}^{n} \psi_{i+s}^{+}\left(q^{m}\right) \psi_{i+s}\left(p^{j}\right)=0,
$$

которое эквивалентно (34).

Для $L \in \mathscr{E}_{k+1, n}$ уравнение (34) совпадает с соотношением дуальности (3) между решениями уравнений, отвечающих операторам $L=L_{E}$ и $\varepsilon L_{G}^{\sigma} \varepsilon^{-1}$. Часть (ii) теоремы 1.4 доказана.

Доказательство теоремы 1.6. Прямое спектральное преобразование (20) для коммутирующих разностных операторов строится аналогично построению прямого спектрального преобразования для периодических операторов. Напомним кратко основные шаги этого построения.

Обозначим через $\mathscr{D}_{k+1}$ аффинное пространство строго треугольных (не обязательно периодических) операторов порядка $k+1$ с нормированным на единицу старшим коэффициентом. Рассмотрим коммутирующую пару операторов $L \in \mathscr{D}_{k+1}$ и $K \in \mathscr{D}_{n-k-1},[L, K]=0$. Ограничение оператора $K$ на $(k+1)$-мерное пространство решений уравнения $(L-E) \psi=0$ задает конечномерный линейный оператор $K(E)$.

Лемма 4.4 [15]. Если $n u k+1$ взаимно просты, то характеристическое уравнение оператора $K(E)$ имеет вид

$$
\mathscr{R}(\kappa, E)=\kappa^{k+1}-E^{n-k-1}+\sum_{(i, j) \in I} \rho_{i j} \kappa^{i} E^{j}=0,
$$

где суммирование берется по парам неотрицательных индексов $(i, j)$, таким, что $0<i(n-k-1)+j(k+1)<(n-k-1)(k+1)$. Для коммутирующих операторов общего положения (для которых спектральная кривая $\Gamma$, заданная уравнением (36), является гладкой) совместная собственная функция $\psi$ операторов $L, K$, $L \psi=E \psi, K \psi=\kappa \psi,(\kappa, E) \in \Gamma$, является функиией Бейкера-Ахиезера.

Число коэффициентов в уравнении $(36)$ равно $(k+2)(n-k) / 2-2$. Для общих значений этих коэффициентов соответствующая кривая $Г$ является гладкой кривой рода $g=k(n-k-2) / 2$. Две отмеченные точки на $\Gamma-$ это точка бесконечности $p_{+}$, в которой обе функции $E$ и $\kappa$ имеют полюсы порядков $k+1$ и $n-k-1$ соответственно, и точка $p_{-}$, в которой обе функции обращаются в нуль. Если кривая Г гладкая, то кольцо $\mathscr{A}_{+}$мероморфных функций на $\Gamma$, имеющих полюс в точке $p_{+}$и равных нулю в точке $p_{-}$, порождено функциями $E$ и $\kappa$. Если $2 k+2<n$, то функция $E$ может быть охарактеризована как образующая кольца $\mathscr{A}_{+}$, имеющая полюс минимальной степени в точке $p_{+}$. Этим 
условием она определяется однозначно с точностью до умножения на константу, $E^{\prime}=c^{k+1} E$. Вторая образующая $\kappa$ этого кольца единственна с точностью до преобразований

$$
\kappa^{\prime}=c^{n-k} \kappa-\sum_{s=1}^{[n /(k+1)]-1} \alpha_{s} E^{s},
$$

которые сохраняют порядок ее полюса в $p_{+}$и форму уравнения $(36)$.

Как уже отмечалось выше, коэффициенты коммутирующих операторов взаимно простых порядков являются квазипериодическими функциями. По предположению теоремы оператор $L$ является $n$-периодическим, т. е. $\left[L, T^{-n}\right]=0$. Это означает, что кольцо $\mathscr{A}_{+}$содержит функцию $w$ с полюсом порядка $n$ в точке $p_{+}$и нулем порядка $n$ в точке $p_{-}$. Поскольку $\mathscr{A}_{+}$порождено функциями $E$ и $\kappa$, функция $w$ имеет следующее разложение по образующим:

$$
w=\kappa E-e \kappa+Q(E),
$$

где $e$ - константа, а $Q$ является полиномом степени $d$, таким, что $d(k+1)<n$ (напомним, что по предположению теоремы $2(k+1)<n$ ), обращающимся в нуль при $E=0$.

Из равенства (37) следует, что функция $w$ равна $\mu:=Q(e)$ во всех нулях $p^{j}$ функции $E-e$. В общем положении эти нули простые. Следовательно, значения функции Бейкера-Ахиезера в этих точках дает базис решений уравнения $(L-e) \Psi=0$, имеющих одинаковый множитель монодромии $\mu$.

Замечание 4.5. Предположение, что точки $\left\{p^{j}\right\}$ являются набором $k+1$ несовпадающих точек, является точным значением выражения «общего положения», фигурировавшего в формулировке теоремы.

Умножение функций $E$ and $\kappa$ на константу $c$ соответствует скейлинговому преобразованию (13) операторов. Подходящим выбором параметра $c$ константа $e$ в равенстве (37) может быть преобразована к $e=-1$. Следовательно, оператор $L$ с точностью до скейлингового преобразования является суперпериодическим. Первое утверждение теоремы доказано.

Для доказательства второго утверждения достаточно заметить, что полином $Q(E)-Q(e)$ делится на $E-e$, т. е. существует единственный полином $P(E)$ без свободного члена, $P(0)=0$, такой, что $Q(E)-\mu=(E-e)\left(P(E)+e^{-1} \mu\right)$. Тогда из уравнения (37) следует равенство

$$
w(p)-\mu=(E(p)-e)\left(\lambda(p)+e^{-1} \mu\right), \quad \lambda(p)=\kappa+P(E) .
$$

Последнее равенство означает, что оператор $\mathscr{L}=K+P(L)$ является суперпериодическим с точностью до скейлингового преобразования. Теорема доказана.

Замечание 4.6. На протяжении всей работы мы рассматривали в основном операторы с комплексными коэффициентами, однако все конструкции и результаты работы допускают простую вещественную редукцию. Если коэффициенты оператора $L$ вещественны, то вещественны и коэффициенты характеристического полинома. Следовательно, комплексное сопряжение задает антиголоморфную инволюцию $\tau$ соответствующих спектральных кривых. Из уравнения $(22)$ следует, что дивизор полюсов $D$ инвариантен относительно антиинволюции $\tau(D)=D$. Следовательно, прямое и обратное спектральные преобразования устанавливают взаимно однозначное соответствие между операторами общего 
положения с вещественными коэффициентами и открытой частью пространства расслоения над вещественными спектральными кривыми, слоем которого является вещественная часть якобиана кривой. Заметим, что расслоение над пространством $M$-кривых, слоем которого является вещественная часть якобиана (дивизоры $D$, описанные в лемме 2.8), соответствует операторам $L$ со знакоопределенным старшим коэффициентом $a_{j}^{1}$.

\section{ЛитеРАТУРА}

[1] I. Assem, C. Reutenauer, D. Smith, Friezes, Adv. Math., 225:6 (2010), 3134-3165.

[2] F. Bergeron, C. Reutenauer, SLk-tilings of the plane, Illinois J. Math., 54:1 (2010), 263-300.

[3] J. H. Conway, H. S. M. Coxeter, Triangulated polygons and frieze patterns, Math. Gaz., 57:400 (1973), 87-94.

[4] H. S. M. Coxeter, Frieze patterns, Acta Arith., 18 (1971), 297-310.

[5] D. Eisenbud, S. Popescu, The projective geometry of Gale transform, J. Algebra, 230:1 (2000), 127-173.

[6] V. Fock, A. Marshakov, Loop groups, clusters, dimers and integrable systems, http:// arxiv.org/abs/1401.1606.

[7] D. Gale, Neighboring vertices on a convex polyhedron, in: Linear Inequalities and Related Systems, Annals of Math. Studies, vol. 38, Princeton University Press, Princeton, NJ, 1956, 255-263.

[8] C. F. Gauss, Pentagramma Mirificum. in: Werke, Bd. III, 481-490; Bd. VIII, 106-111.

[9] I. M. Gelfand, R. D. MacPherson, Geometry in Grassmanianns and generalization of the dilogarithm, Adv. in Math., 44:3 (1982), 279-312.

[10] M. Gekhtman, M. Shapiro, S. Tabachnikov, A. Vainshtein, Higher pentagram maps, weighted directed networks, and cluster dynamics, Electron. Res. Announc. Math. Sci., 19 (2012), 1-17.

[11] M. Kapranov, Chow quotients of Grassmanianns, I, in: "I. M. Gelfand Seminar", Advances in Soviet Math., vol. 16, Part 2, Amer. Math. Soc., Providence, RI, 1993, 29-110.

[12] B. Khesin, F. Soloviev, Integrability of higher pentargam maps, Math. Ann., 357:3 (2013), 1005-1047.

[13] B. Khesin, F. Soloviev, The geometry of dented pentagram maps, http://arxiv.org/ abs/1308.5363.

[14] И. М. Кричевер, Интегрирование нелинейных уравнений методами алгебраической геометрии, Функц. анализ и его прил., 11:1 (1977), 15-31.

[15] И. М. Кричевер, Алгебраические кривые и линейне разностные уравнения, УМН, 33:4 (1979), 215-216.

[16] И. М. Кричевер, Нелинейные уравнения и эллиптические кривые, в кн.: Итоги науки и техники, Современные проблемы математики, т. 23, ВИНИТИ, 1983, 79 139.

[17] И. М. Кричевер, С. П. Новиков, Двумеризованная цепочка Тодь, коммутирующие разностные операторы и голоморфные расслоения, УМН, 58:3 (2003), 51-88.

[18] G. M. Beffa, On integrable generalizations of the pentagram map, Int. Math. Res. Not., No. 12 (2015), 3669-3693; http://arxiv.org/abs/1303.4295.

[19] S. Morier-Genoud, Arithmetics of 2-friezes, J. Algebraic Combin., 36:4 (2012), 515539 .

[20] S. Morier-Genoud, V. Ovsienko, S. Tabachnikov, 2-Frieze patterns and the cluster structure of the space of polygons, Ann. Inst. Fourier, 62:3 (2012), 937-987. 
[21] S. Morier-Genoud, V. Ovsienko, R. E. Schwartz, S. Tabachnikov, Linear difference equations, frieze patterns and combinatorial Gale transform, Forum Math. Sigma, 2 (2014), e22; http://arxiv.org/abs/1309.3880.

[22] S. Morier-Genoud, V. Ovsienko, S. Tabachnikov, $S L_{2}(\mathbb{Z})$-tiling of the torus, CoxeterConway friezes and Farey triangulations, http://arxiv.org/abs/1402.5536.

[23] D. Mumford, An algebro-geometric construction of commuting operators and of solutions to the Toda lattice equation, Korteweg-de Vries equation and related nonlinear equations, in: Proc. Int. Symp. Algebraic Geometry (Kyoto, 1977), Kinokuniya Book Store, Tokyo, 1978, 115-153.

[24] V. Ovsienko, R. Schwartz, S. Tabachnikov, The pentagram map: A discrete integrable system, Comm. Math. Phys., 299:2 (2010), 409-446.

[25] V. Ovsienko, R. Schwartz, S. Tabachnikov, Liouville-Arnold integrability of the pentagram map on closed polygons, Duke Math. J., 162:12 (2013), 2149-2196.

[26] V. Ovsienko, S. Tabachnikov, Coxeter's frieze patterns and discretization of the Virasoro orbit, http://arxiv.org/abs/1312.3021.

[27] J. Propp, The combinatorics of frieze patterns and Markoff numbers, http://arxiv. org/abs/math/0511633.

[28] R. Schwartz, Discrete monodromy, pentagrams, and the method of condensation, J. Fixed Point Theory Appl., 3:2 (2008), 379-409.

[29] R. Schwartz, The pentagramm map, Experiment. Math., 1:1 (1992), 71-81.

[30] F. Soloviev, Integrability of the pentagram map, Duke. Math. J., 162:15 (2013), 28152853.

[31] A. Zabrodin, Discrete Hirota's equation in quantum integrable models, Internat. J. Modern Phys. B, 11:26-27 (1997), 3125-3158.

Department of Mathematics, Columbia University

Национальный исследовательский университет

Поступила в редакцию «Высшая школа экономики», факультет математики

Институт проблем передачи информации

им. А. А. Харкевича РАН

Институт теоретической физики

им. Л. Д. Ландау РАН

e-mail: krichev@math.columbia.edu 